\title{
Identification of a novel mutation of the PRKAR1A gene in a patient with Carney complex with significant osteoporosis and recurrent fractures
}

\author{
Labrini Papanastasiou, ${ }^{1}$ Stelios Fountoulakis, ${ }^{1}$ Nikos Voulgaris, ${ }^{1}$ Theodora Kounadi, ${ }^{1}$ \\ Theodosia Choreftaki, ${ }^{2}$ Akrivi Kostopoulou, ${ }^{2}$ George Zografos, ${ }^{3}$ \\ Charalampos Lyssikatos, ${ }^{4}$ Constantine A. Stratakis, ${ }^{4}$ George Piaditis ${ }^{1}$
}

${ }^{1}$ Department of Endocrinology and Diabetes Center, ${ }^{2}$ Department of Pathology, ${ }^{3}$ Department of Surgery; "G. Gennimatas" General Hospital, Athens, Greece; ${ }^{4}$ Section on Endocrinology \& Genetics, Program on Developmental Endocrinology \& Genetics, National Institute of Child Health and Human Development, National Institutes of Health, Bethesda, USA

\begin{abstract}
OBJECTIVE: Carney complex (CNC) is a rare autosomal dominant multiple neoplasia syndrome characterized by the presence of endocrine and non-endocrine tumors. More than 125 different germline mutations of the protein Kinase A type 1- $\alpha$ regulatory subunit (PRKAR1A) gene have been reported. We present a novel $P R K A R 1 A$ gene germline mutation in a patient with severe osteoporosis and recurrent vertebral fractures. DESIGN: Clinical case report. CASE REPORT: A 53-year-old male with a medical history of surgically removed recurrent cardiac myxomas was evaluated for repeated low-pressure vertebral fractures and severe osteoporosis. Physical examination revealed spotty skin pigmentation of the lower extremities and papules in the nuchal and thoracic region. The presence of hypercortisolism due to micronodular adrenal disease and the history of cardiac myxomas suggested the diagnosis of CNC; the patient underwent detailed imaging investigation and genetic testing. METHODS: Standard imaging and clinical testing; DNA was sequenced by the Sanger method. RESULTS: Sequence analysis from peripheral lymphocytes DNA revealed a novel heterozygous point mutation at codon 172 of exon 2 (c.172G $>$ T) of the PRKAR1A gene, resulting in early termination of the PRKAR1A transcript [p.Glu58Ter (E58X)]. CONCLUSION: We report a novel point mutation of the PRKAR1A gene in a patient with CNC who presented with significant osteoporosis and fractures. Low bone mineral density along with recurrent myxomas should point to the diagnosis of $\mathrm{CNC}$.
\end{abstract}

Key words: Carney complex, Hypercortisolism, Osteoporosis, PRKAR1A gene, PPNAD

Address for correspondence:

Labrini Papanastasiou, MD, PHD, Department of Endocrinology

and Diabetes Center, General Hospital "G. Gennimatas",

154 Mesogion Avenue, 11527 Athens, Greece;

Tel.: +30 2107768283, Fax: +30 2107779146,

E-mail: linapapan@yahoo.gr

Received: 19-07-2015, Accepted: 09-09-2015

\section{INTRODUCTION}

Carney complex $(\mathrm{CNC})$ is a rare disease with an autosomal dominant inheritance characterized by the presence of myxomas, spotty skin pigmentation and endocrine overactivity. ${ }^{1} \mathrm{CNC}$ is associated with a 
variety of endocrine and non-endocrine abnormalities, the most frequent being primary pigmented nodular adrenocortical disease (PPNAD) that usually leads to adrenocorticotropin (ACTH)-independent Cushing's syndrome (CS). Other common endocrine abnormalities include cystic or nodular thyroid disease in about $75 \%$ of patients, subtle hyperprolactinemia (64\%), acromegaly (in up to $15 \%$ ) and large-cell calcifying Sertoli tumors ( $41 \%$ of affected males). Non-endocrine abnormalities include skin myxomas $(80 \%$ of patients), lentiginosis (80\%), breast fibroadenomas or myxomas $(50 \%)$, cardiac myxomas $(20-40 \%)$, cutaneous myxomas (20-30\%) and psammomatous melanotic schwannomas $(8 \%){ }^{2}$

$\mathrm{CNC}$ is a genetically heterogeneous disease, with linkage analysis so far identifying two independent loci [17q22-24 (CNC1), 2p16 (CNC2)]; a third locus was erroneously linked to $\mathrm{CNC}$, reflecting the oddity of a single family with myosin mutations and the concurrent occurrence of myxomas. ${ }^{3-5}$ The most common genetic cause of CNC is a defect in the PRKAR1A gene (at the CNC1 locus). PRKAR1A encodes for the 1- $\alpha$ regulatory subunit $(\mathrm{RI}-\alpha)$ of protein Kinase A (PKA) and functions as a tumor suppressor gene (TSG). ${ }^{6}$ Heterozygous inactivating PRKARIA mutations lead to CNC with a penetrance close to $98 \%$ by the age of 50 years; these mutations have been reported in $73 \%$ of CNC patients. ${ }^{2,7,8}$ The majority of these mutations result in frame-shift, nonsense or splice site variants that lead to premature stop-codon generation. ${ }^{9}$ Mutant mRNA is unstable and degraded by nonsense-mediated mRNA decay (NMD). This leads to the loss of the mutant protein and a $50 \%$ reduction of the total RI- $\alpha$ protein levels, since only the wild type allele is translated. ${ }^{6} \mathrm{RI}-\alpha$ protein reduction stimulates protein kinase $\mathrm{A}$ (PKA) activity by cyclic adenosine monophosphate (cAMP) thus interfering in the regulation of cell glucose and lipid metabolism pathways. ${ }^{2}$ Until recently, about $750 \mathrm{CNC}$ patients have been diagnosed worldwide by the National Institute of Health, the Mayo Clinic (U.S.A), the Cochin Hospital (France) and elsewhere, with more than 125 PRKAR1A gene mutations identified to date (online database: http:prkarla.nichd.nih.gov). ${ }^{9}$ Despite the genetic heterogeneity and the large number of PRKAR $1 A$ mutations spread along the length of the gene, no direct correlation between all PRKARIA mutations and the various $\mathrm{CNC}$ phenotypes has yet been established. However, recent data report potential associations between specific mutations and $\mathrm{CNC}$ manifestations. ${ }^{2,10-13}$

In this article we present a $\mathrm{CNC}$ case with a novel germline PRKARIA mutation that was diagnosed after recurrent vertebral fractures presumably due to hypercortisolemia and we provide a brief review of the existing literature concerning genotype and phenotype associations in CNC.

\section{CASE REPORT}

A 53-year-old male (weight $69 \mathrm{~kg}$, height $1.72 \mathrm{~m}$, blood pressure $110 / 70 \mathrm{mmHg}$ ) was admitted to our Department for evaluation of deteriorating severe osteoporosis (left femoral neck T-score: -4.2 , right femoral neck T-score: -4.0, L2-L4 T-score: -5.2), resulting in low-pressure fractures of lumbar spine vertebrae and muscle weakness. Radiologic imaging revealed T8-T10 and L3-L5 vertebral compression fractures. The patient had a history of prior T12-L2 spondylodesis due to a low-pressure L1 vertebra osteoporotic fracture and was treated with denosumab and calcium (500mg bd) for two years without any bone mass improvement. Over the last three decades he had undergone three cardiac operations for recurrent atrial and ventricular peduncular myxomas (left atrium and right ventricle), with his last serial echocardiography not revealing the presence of any myxoma. He had also undergone polipectomy following two episodes of intestinal blood loss. No family history of endocrine or non-endocrine tumors was identified.

On admission he had muscle weakness and incapacitating back pain. Physical examination revealed spotty skin pigmentation (lentigines) on both legs and two $2 \mathrm{~cm}$ brownish papules on the preauricolar and lower right thoracic region, consistent with cutaneous myxomas. Hormonal work-up revealed hypercortisolism [(08.00 morning cortisol levels: 389 nmol/L (NR: 138-690 nmol/L), adrenocorticotropic hormone (ACTH): $5.5 \mathrm{pg} / \mathrm{ml}$ (NR: 9-52 pg/ml), urinary cortisol concentration (UFC): $210 \mu \mathrm{g} / 24 \mathrm{~h}$ (NR: 20-90 $\mu \mathrm{g} / 24 \mathrm{~h}$ ), cortisol levels following a low-dose dexamethasone suppression test (LDDST): $412 \mathrm{nmol} / \mathrm{L}$ and paradoxical increase of UFC $(303 \mu \mathrm{g} / 24 \mathrm{~h})$ following dexamethasone administration]. Secondary 
hyperparathyroidism [parathyroid hormone (PTH): 140 $\mathrm{pg} / \mathrm{ml}$ (NR: $11-62 \mathrm{pg} / \mathrm{ml}$ ), corrected plasma calcium: $8.6 \mathrm{mg} / \mathrm{dl}$, plasma phosphorus: $3 \mathrm{mg} / \mathrm{dl}, 25-\mathrm{OHD}_{3}: 15$ $\mathrm{ng} / \mathrm{ml}$ ] was also evident. Prolactin [(PRL: $13.9 \mathrm{ng} / \mathrm{ml}$ (NR: 3.46-19.4)], free thyroxin [(FT4: $14.8 \mathrm{pmol} / \mathrm{L}$ (NR: 9.01-21 pmol/L)], thyrotropin [(TSH: $1.6 \mu \mathrm{IU} /$

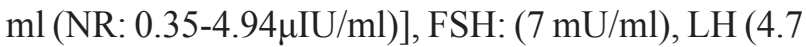
$\mathrm{mU} / \mathrm{ml}$ ) and SHBG levels [38nmol/L (NR: 30-100 $\mathrm{nmol} / \mathrm{L})$ ] were normal. Growth hormone (GH: 2.9 $\mathrm{ng} / \mathrm{ml}$ ) and $\mathrm{GH}$ response to a $75 \mathrm{gr}$ oral glucose load (nadir of GH: $1 \mathrm{ng} / \mathrm{ml}$ ) were also normal. Testosterone [1.7 ng/ml (NR: 2.67-10.12 ng/ml)], DHEA-S [468 $\mathrm{ng} / \mathrm{ml}$ (NR: $518-4707 \mathrm{ng} / \mathrm{ml}$ )] and insulin-like growth factor-1(IGF-1) levels [117 ng/ml (NR for sex and age: $180-406 \mathrm{ng} / \mathrm{ml})$ ] were decreased.

Adrenal computed tomography (CT) showed micronodular adrenal disease (Figure 1). Ultrasonog-

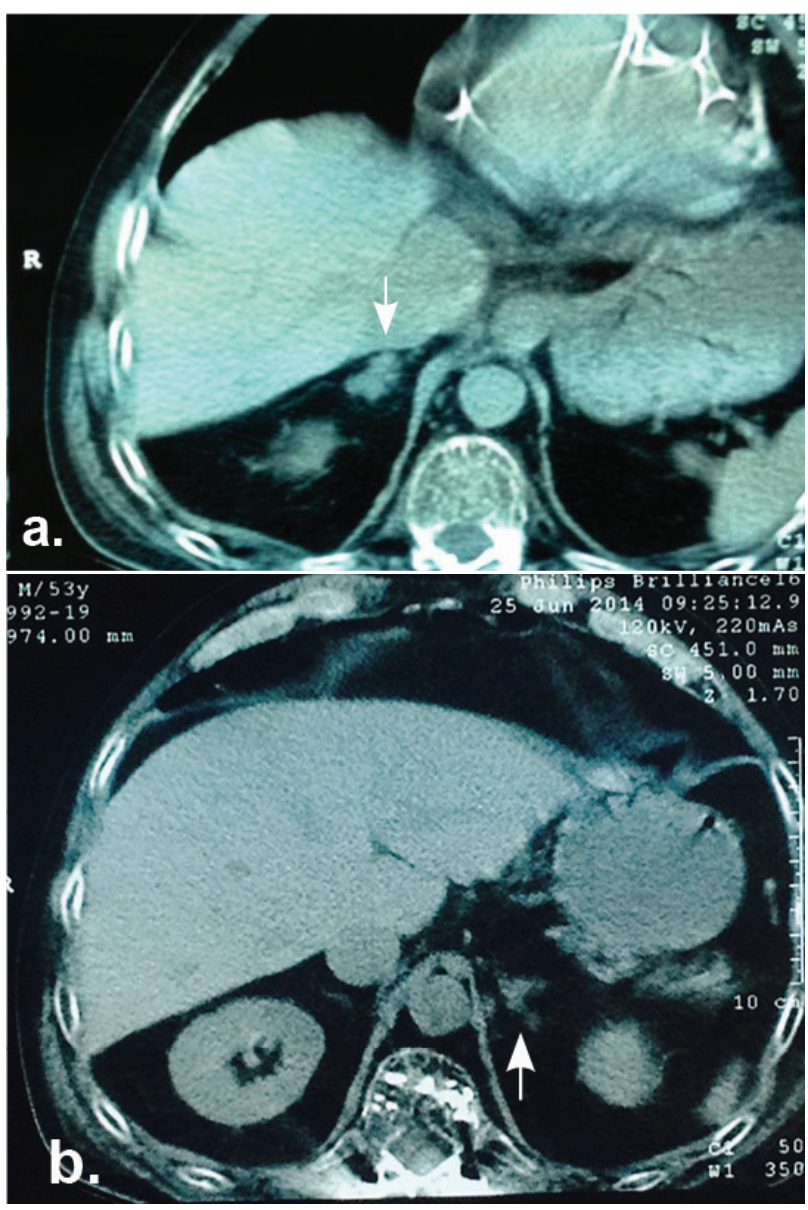

Figure 1. Adrenal computed tomography revealing bilateral nodularity of the adrenal glands. raphy demonstrated multiple thyroid nodules and multiple microcalcifications of the testes, whereas echocardiography did not reveal any recurrent cardiac myxomas. Pituitary magnetic resonance imaging (MRI) was also normal.

Denosumab was discontinued and the patient was prescribed ketoconazole $(400 \mathrm{mg} /$ day) and metopyrone $(1.5 \mathrm{gr} /$ day) while on the waiting list for bilateral adrenalectomy. Surgically removed adrenal glands appeared with multiple pigmented nodules (Figure 2 ) and histopathology revealed multiple, $0.1-0.5 \mathrm{~cm}$ sized, pigmented nodules surrounded by atrophic cortex (Figure 3). Histological results were consistent with the diagnosis of primary pigmented nodular adrenocortical disease (PPNAD). Most of the nodule cells were large and globular with granular eosinophilic cytoplasm that included lipofuscin. Myxomatous areas were also detected within the nodules (Figure 3).

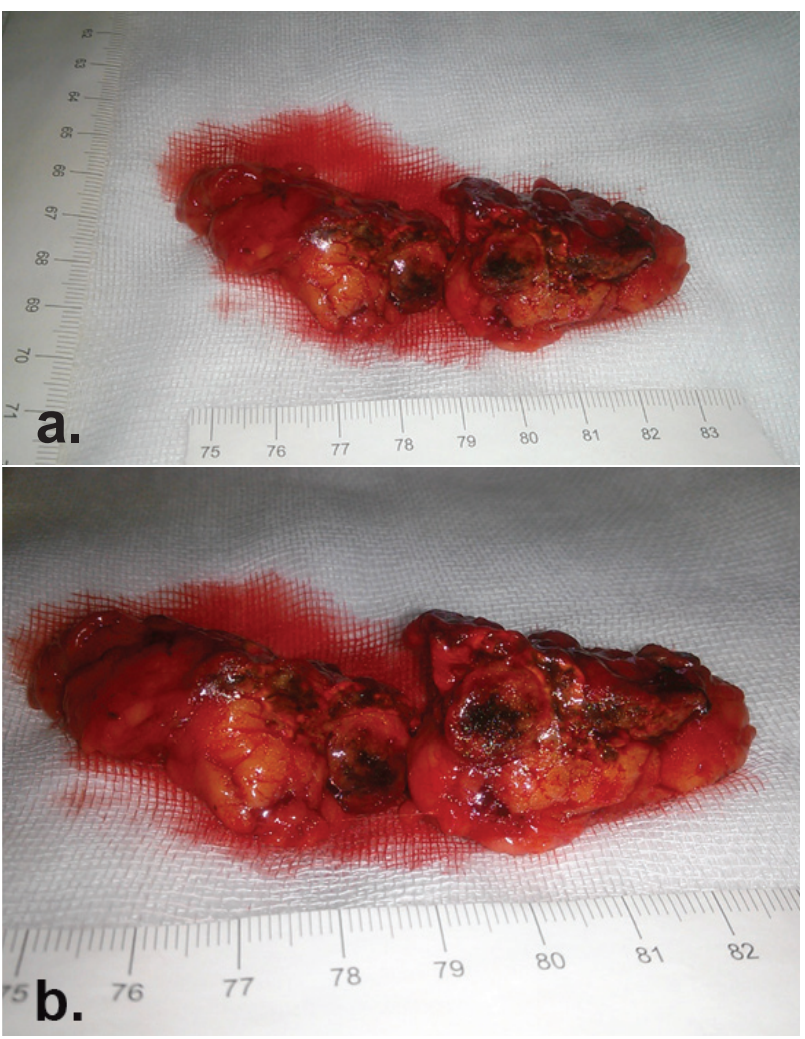

Figure 2. Macroscopic appearance of the surgically removed PPNAD adrenal gland. Multiple pigmented micronodules can be seen in the cross-section. 

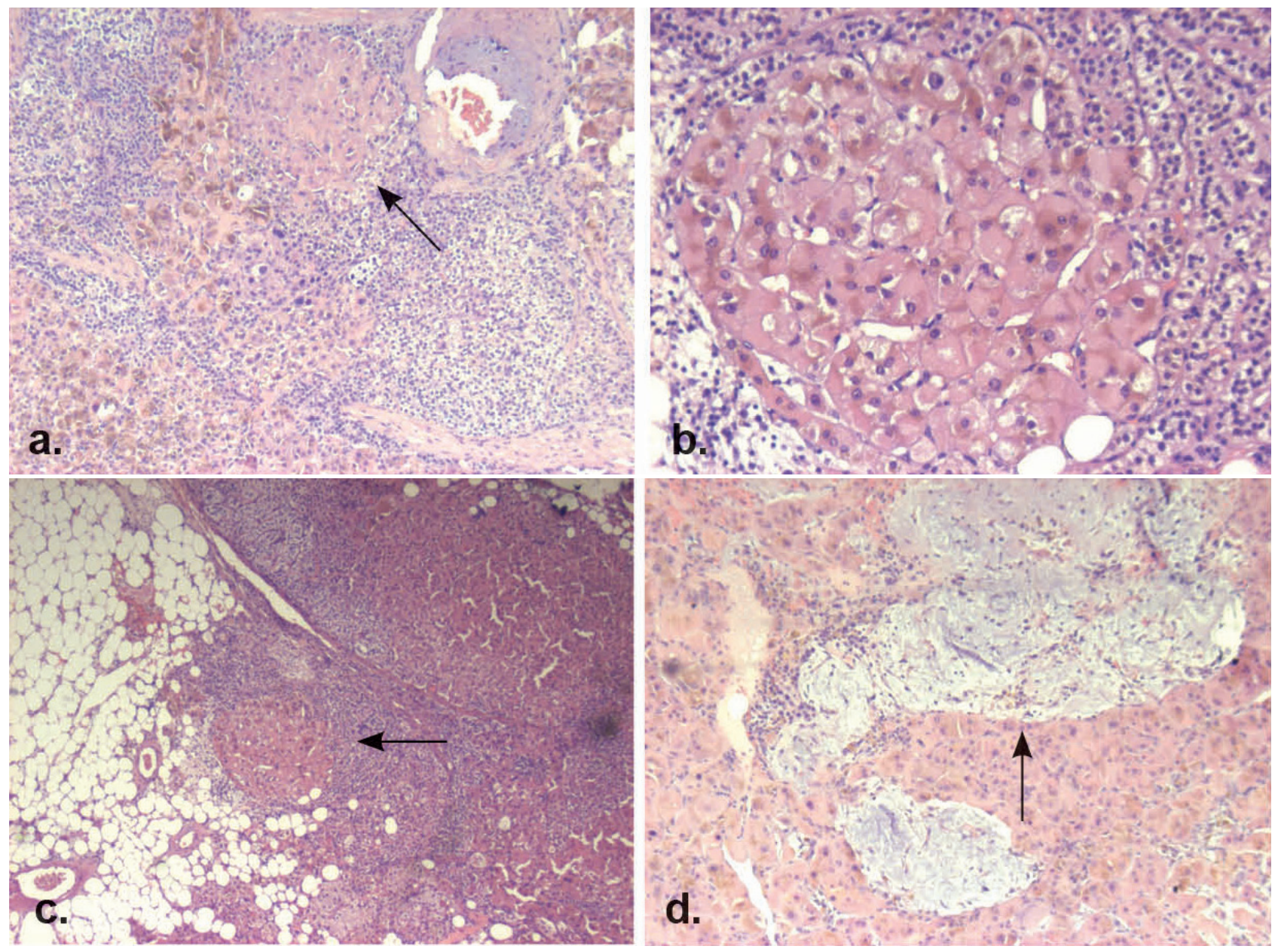

Figure 3. Pathology of the adrenal glands. a. Classic appearance of PPNAD (x50). b. Nodular cortical cells containing large nucleus and eosinophilic cytoplasm with lipofuscin (x100). c. Expansion of cortical nodules to the adjacent adipose tissue (x25). d. Myxomatous areas within the nodule; hematoxylin and eosin stain (x50).

\section{SEQUENCING ANALYSIS}

A peripheral blood sample was drawn after obtaining informed consent from the patient. DNA was extracted from peripheral leucocytes and subjected to polymerase chain reaction (PCR), followed by bidirectional DNA sequence analysis of the PRKARIA gene, performed as described previously. ${ }^{6,14}$ The nucleotide sequence was compared with the published cDNA PRKAR1A sequence. A novel c.172G $>$ T heterozygous point mutation at codon 172 of exon 2 was identified that resulted in direct stop-codon generation and in early termination of PRKARIA transcript (Figure 4).

\section{DISCUSSION}

$\mathrm{CNC}$ is a rare endocrine syndrome which is most frequently caused by PRKARIA gene mutations. We report a novel germline nonsense mutation of the PRKAR1A gene in a patient with clinical features as well as laboratory and histological findings of CNC. Our patient had developed a wide heterogeneous spectrum of CNC-associated manifestations including hypercortisolism with PPNAD and secondary osteoporosis, skin lentigines and myxomas, multiple recurrent cardiac myxomas and nodular thyroid disease. We also found a paradoxical increase of cortisol secretion after dexamethasone administration, which is characteristic of CNC and particularly useful for the diagnosis of patients with normal baseline cortisol levels and subclinical or cyclic CS. ${ }^{15}$

Osteoporotic bone changes are often found in $\mathrm{CNC}$ patients. Glucocorticoids excess accelerates bone resorption and reduces bone formation by direct action 

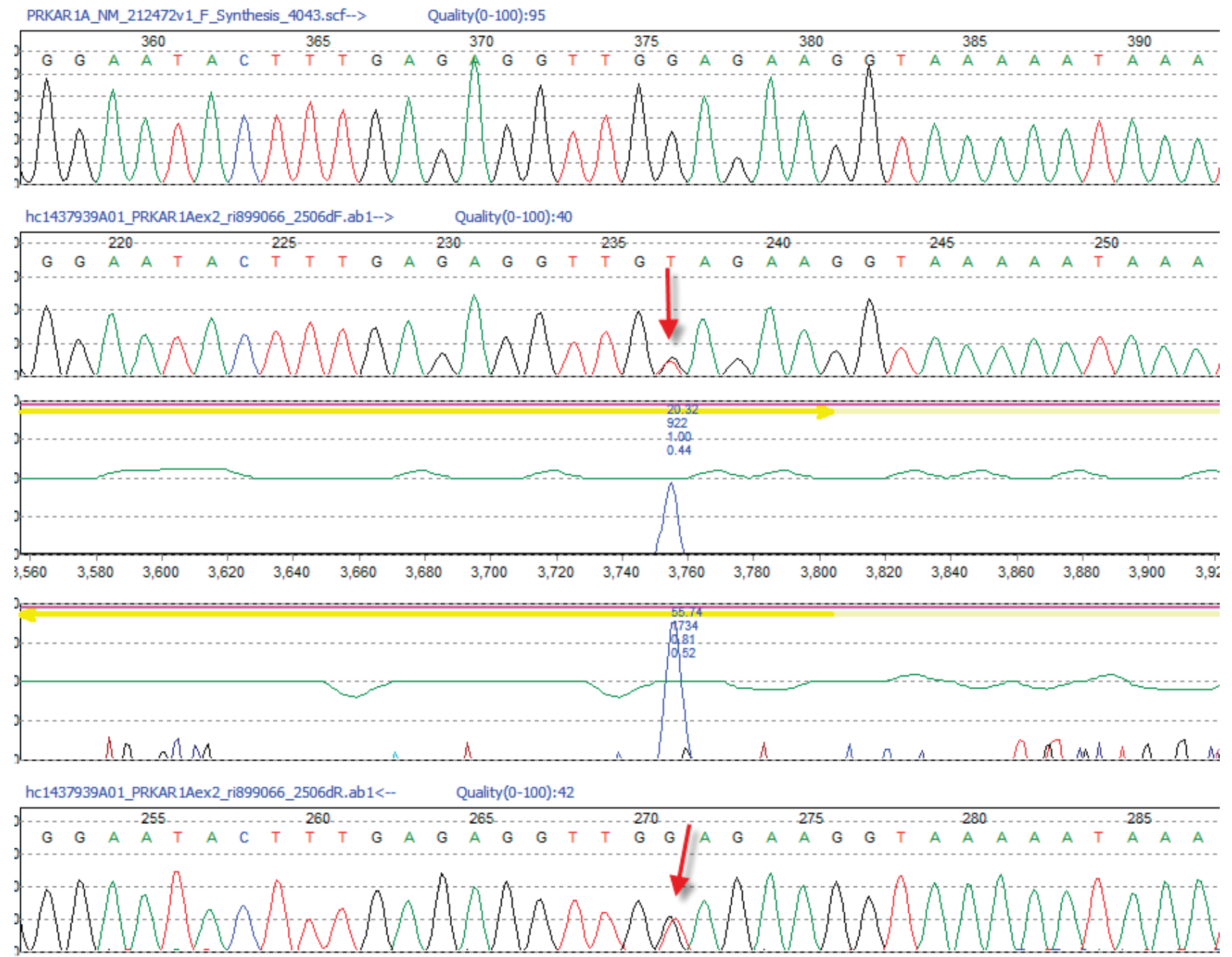

PRKAR1A_NM_212472v1_R_Synthesis_4043.scf<-- Quality(0-100):95

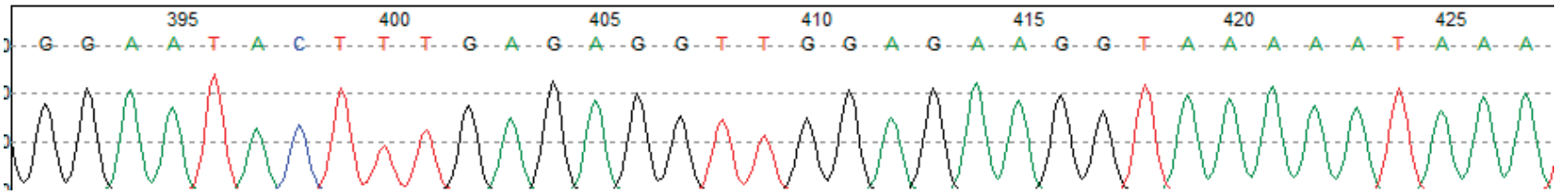

Figure 4. Direct sequencing of leukocytes RT-PCR products demonstrating the p.E58Xc.172 GAG>TAG mutation of the PRKAR1A gene.

on bone cells. In addition, glucocorticoids decrease intestinal calcium absorption by opposing the action of vitamin D and by decreasing the expression of calcium channels in the duodenum leading to secondary hyperparathyroidism. ${ }^{16}$ They also inhibit IGF-1and serum testosterone production (as observed in our patient) ${ }^{16,17}$ Apart from the secondary osteoporosis due to PPNAD-associated cortisol hypersecretion, the osteogenic potential may also be influenced by PRKARIA gene ablation that can interfere with sign- aling pathways which are necessary for osteoblast differentiation, as shown in experimental data from mouse and human cell lines..$^{18}$ The osteoporotic bone changes, fractures and secondary hyperparathyroidism that were found in our $\mathrm{CNC}$ patient indicated the long duration of the disease.

Recurrent cardiac myxomas occur at a much younger age in CNC patients compared to sporadically occurring myxomas. ${ }^{19}$ Interestingly, up to $30 \%$ of non-CNC myxomas can also bear PRKARIA muta- 
tions, suggesting a potential role of the gene and the PKA pathway in the development of both syndromic and isolated cardiac myxomas. ${ }^{20}$ The PRKARlA mutation found in our patient caused lack of mutant protein detection due to degradation, similarly to other PRKAR1A mutations reported so far. Although genetic screening was not performed in the close relatives of our patient, the absence of any characteristics of the disease suggests that this mutation is a de novo one. This finding is in accordance with previous studies that described de novo mutation at a high frequency $(85 \%)$ in sporadic cases with $\mathrm{CNC}^{6}$

In contrast to RET mutations in MEN2 syndrome, it is widely accepted that $P R K A R 1 A$ gene mutations do not appear to correlate consistently with a specific clinical phenotype. However, data overall indicate that CNC patients bearing PRKAR1A mutations (CNC1) have more severe disease with earlier presentation and higher frequency of myxomas, thyroid and gonadal tumors, schwannomas and lentigines compared to PRKAR1A negative patients with a mutation mapped in the $2 \mathrm{p} 16, \mathrm{CNC} 2$ locus. ${ }^{2,414}$ In addition, a small number of PRKARIA missense mutations whose mRNA escape from NMD and express R I- $\alpha$ mutant proteins are associated with a more severe phenotype. ${ }^{21,22}$ Two PRKAR1A mutations (a deletion c.709-7del6 and a mutation in the initiation codon of PRKAR1A, M1V c.1A>G/p.M1Vsubstitution) are associated with low-penetrance, early life isolated PPNAD and CS. ${ }^{23,24}$ Moreover, in some cases of isolated PPNAD, a small intronic deletion in PRKAR1A has been associated with a lower penetrance and mild phenotype. ${ }^{25}$ In general, patients with exonic PRKAR1A mutations seem to present at a younger age and to manifest cardiac myxomas, lentigines, schwannomas and acromegaly more often compared to patients with intronic ones.

In the study of Salpea et al, a significant number (21.6\%) of CNC patients had haploinsufficiency due to large 17q24.2-q24.3 deletions surrounding the PRKARIA gene. ${ }^{10}$ These deletions were not detected by Sanger sequencing (PRKAR1A mutation-negative) but with array-based comparative genomic hybridization (array-CGH). It is worth noting that apart from the usual CNC-related manifestations, some of these patients shared skeletal abnormalities and global developmen- tal delay. Moreover, the average age of presentation was younger (14 years) and morbidity more severe than in typical PRKARIA mutation-positive CNC patients. In addition, in a family of CNC patients, a spectrum of disease including adrenal carcinoma due to a $\mathrm{S} 147 \mathrm{G}$ point mutation in the PRKAR1A gene that escapes NMD, was identified. This mutation led to decreased cAMP and catalytic subunit binding by $\mathrm{R}$ I- $\alpha$ and increased PKA activity in vitro. ${ }^{11}$

Lately, genes coding for other PKA subunits have been identified as being responsible for CNC-linked phenotypes (but not the full syndrome). Duplication of the main catalytic subunit of the cAMP-dependent PKA, $\mathrm{C} \alpha(P R K A C A)$, may result in Cushing syndrome caused by bilateral micronodular adrenal hyperplasia. ${ }^{12}$ In a single patient, triplication of the catalytic subunit $C \beta$ (the $P R K A C B$ gene) was associated with skin pigmentation, acromegaly and myxomas. ${ }^{13}$

All the aforementioned genetic data could have implications for our understanding of the $\mathrm{CNC}$ phenotype and, more importantly, for counseling CNC patients. Genetic analysis is a powerful tool aiding the investigation, confirmation and early identification of tumors and endocrine disorders related to CNC. Younger age of onset and potential diverse prognosis according to the genotype could help us properly counsel patients' family members and descendants. Even though there is yet no obvious and straightforward correlation between genotype findings and the clinical characteristics of the disease, there is growing evidence that a genotype-phenotype association is plausible for at least certain PRKARIA defects.

In conclusion, we have identified a novel PRKARIA nonsense mutation in a sporadic case of CNC. The data add to what we know about PRKARIA and human disease. Recognizing CNC is important for identifying early and addressing promptly the many associated comorbidities, including acromegaly, atrial fibrillation and stroke due to atrial myxomas, diabetes mellitus, hypertension, osteoporosis and fractures due to hypercortisolism, and the risk of various tumors and certain cancers. PRKAR1A mutation analysis should be undertaken in suspected cases of CNC in order to confirm the diagnosis and provide close monitoring and follow-up. 


\section{ACKNOWLEDGEMENTS/FUNDING}

This work was supported by the Intramural Research Program (IRP) of the Eunice Kennedy Shriver National Institute of Child Health \& Human Development (NICHD), National Institutes of Health (NIH), Bethesda, MD 20892, USA.

\section{CONFLICT OF INTEREST STATEMENT}

The authors have no conflict of interest to disclose.

\section{REFERENCES}

1. Carney JA, Gordon H, Carpenter PC, et al, 1985 The complex of myxomas, spotty pigmentation, and endocrine overactivity. Medicine (Baltimore) 64: 270-283.

2. Bertherat J, Horvath A, Groussin L, et al, 2009 Mutations in regulatory subunit type $1 \mathrm{~A}$ of cyclic adenosine 5'-monophosphate-dependent protein kinase (PRKAR1A): phenotype analysis in 353 patients and 80 different genotypes. J Clin Endocrinol Metab 94: 2085-2091.

3. Casey M, Mah C, Merliss AD, et al, 1998 Identification of a novel genetic locus for familial cardiac myxomas and Carney complex. Circulation 98: 2560-2566.

4. Stratakis CA, Carney JA, Lin JP, et al, 1996 Carney complex, a familial multiple neoplasia and lentiginosis syndrome. Analysis of 11 kindreds and linkage to the short arm of chromosome 2. J Clin Invest 97: 699-705.

5. Veugelers M, Bressan M, McDermott DA, et al, 2004 Mutation of perinatal myosin heavy chain associated with a Carney complex variant. N Engl J Med 351: 460-469.

6. Kirschner LS, Carney JA, Pack SD, et al 2000 Mutations of the gene encoding the protein kinase A type I-alpha regulatory subunit in patients with the Carney complex. Nat Genet 26: 89-92.

7. Veugelers M, Wilkes D, Burton K, et al, 2004 Comparative PRKAR1A genotype-phenotype analyses in humans with Carney complex and prkarla haploinsufficient mice. Proc Natl Acad Sci U S A 101: 14222-14227.

8. Groussin L, Kirschner LS, Vincent-Dejean C, et al, 2002 Molecular analysis of the cyclic AMP-dependent protein kinase A (PKA) regulatory subunit 1A (PRKAR1A) gene in patients with Carney complex and primary pigmented nodular adrenocortical disease (PPNAD) reveals novel mutations and clues for pathophysiology: augmented PKA signaling is associated with adrenal tumorigenesis in PPNAD. Am J Hum Genet 71: 1433-1442.

9. Horvath A, Bertherat J, Groussin L, et al, 2010 Mutations and polymorphisms in the gene encoding regulatory subunit type 1-alpha of protein kinase A (PRKAR1A): an update. Hum Mutat 31: 369-379.

10. Salpea P, Horvath A, London E, et al, 2014 Deletions of the PRKAR1A locus at 17q24.2-q24.3 in Carney complex: genotype-phenotype correlations and implications for genetic testing. J Clin Endocrinol Metab
99: E183-188.

11. Anselmo J, Medeiros S, Carneiro V, et al, 2012 A large family with Carney complex caused by the S147G PRKAR1A mutation shows a unique spectrum of disease including adrenocortical cancer. J Clin Endocrinol Metab 97: 351-359.

12. Beuschlein F, Fassnacht M, Assié G, et al, 2014 Constitutive activation of PKA catalytic subunit in adrenal Cushing's syndrome. N Engl J Med 370: 1019-1028.

13. Forlino A, Vetro A, Garavelli L, et al, 2014 PRKACB and Carney complex. N Engl J M 370: 1065-1067.

14. Kirschner LS, Sandrini F, Monbo J, et al, 2000 Genetic heterogeneity and spectrum of mutations of the PRKAR1A gene in patients with the carney complex. Hum Mol Genet 9: 3037-3046.

15. Stratakis CA, Sarlis N, Kirschner LS, et al, 1999 Paradoxical response to dexamethasone in the diagnosis of primary pigmented nodular adrenocortical disease. Ann Intern Med 131: 585-591.

16. Canalis 2007, Huybers S, Naber TH, et al, 2007 Prednisolone-induced $\mathrm{Ca} 2+$ malabsorption is caused by diminished expression of the epithelial Ca2+ channel TRPV6. Am J Physiol Gastrointest Liver Physiol 292: G92.

17. MacAdams MR, White RH, Chipps BE, 1986 Reduction of serum testosterone levels during chronic glucocorticoid therapy. Ann Intern Med 104: 648.

18. Zhang M, Manchanda PK, Wu D, et al, 2014 Knockdown of PRKAR1A, the gene responsible for Carney complex, interferes with differentiation in osteoblastic cells. Mol Endocrinol 28: 295-307.

19. Azzam R, Abdelbar A, Yap KH, et al, 2014 Carney complex: fourth time excision of recurrent atrial myxoma via left thoracotomy. BMJ Case Rep 2014 ppi: bcr2013201827.

20. Maleszewski JJ, Larsen BT, Kip NS, 2014 PRKAR1A in the development of cardiac myxoma: a study of 110 cases including isolated and syndromic tumors. Am J Surg Pathol 38: 1079-1087.

21. Greene EL, Horvath AD, Nesterova M, et al, 2008 In vitro functional studies of naturally occurring pathogenic PRKAR1A mutations that are not subject to nonsense mRNA decay. Hum Mutat 29: 633-639.

22. Meoli E, Bossis I, Cazabat L, et al, 2008 Protein kinase A effects of an expressed PRKAR1A mutation associated with aggressive tumors. Cancer Res 68: 3133-3141.

23. Groussin L, Jullian E, Perlemoine K, et al, 2002 Mutations of the PRKAR1A gene in Cushing's syndrome due to sporadic primary pigmented nodular adrenocortical disease. J Clin Endocrinol Metab 87: 4324-4329.

24. Pereira AM, Hes FJ, Horvath A, et al, 2010 Association of the M1V PRKAR1A mutation with primary pigmented nodular adrenocortical disease in two large families. J Clin Endocrinol Metab 95: 338-342.

25. Groussin L, Horvath A, Jullian E, et al, 2006 A PRKAR1A mutation associated with primary pigmented nodular adrenocortical disease in 12 kindreds. J Clin Endocrinol Metab 91: 1943-1949. 